\title{
Anastassios Anastassiadis (éd.), Voisinages fragiles. Les relations interconfessionnelles dans le Sud-Est européen et la Méditerranée orientale 1854-1923 : contraintes locales et enjeux internationaux
}

Athènes, École française d'Athènes, 2013, 336 p.

\section{Bernard Heyberger}

\section{(epenEdition}

Journals

\section{Édition électronique}

URL : http://journals.openedition.org/assr/27272

DOI : $10.4000 /$ assr. 27272

ISSN : $1777-5825$

Éditeur

Éditions de l'EHESS

\section{Édition imprimée}

Date de publication : 1 octobre 2015

Pagination : 241

ISBN : 978-2-7132-2515-4

ISSN : 0335-5985

\section{Référence électronique}

Bernard Heyberger, « Anastassios Anastassiadis (éd.), Voisinages fragiles. Les relations interconfessionnelles dans le Sud-Est européen et la Méditerranée orientale 1854-1923: contraintes locales et enjeux internationaux », Archives de sciences sociales des religions [En ligne], 172 I octobredécembre, mis en ligne le 13 mai 2016, consulté le 24 septembre 2020. URL : http:// journals.openedition.org/assr/27272; DOI : https://doi.org/10.4000/assr.27272

Ce document a été généré automatiquement le 24 septembre 2020.

(C) Archives de sciences sociales des religions 


\section{Anastassios Anastassiadis (éd.), Voisinages fragiles. Les relations interconfessionnelles dans le Sud- Est européen et la Méditerranée orientale 1854-1923 : contraintes locales et enjeux internationaux}

Athènes, École française d'Athènes, 2013, 336 p.

\section{Bernard Heyberger}

\section{RÉFÉRENCE}

Anastassios Anastassiadis (éd.), Voisinages fragiles. Les relations interconfessionnelles dans le Sud-Est européen et la Méditerranée orientale 1854-1923 : contraintes locales et enjeux internationaux, Athènes, École française d'Athènes, 2013, 336 p.

Comme la préface d'Anastassios Anastassiadis le souligne, cet ouvrage issu d'un colloque est structuré autour d'un certain nombre d'idées force, qui amènent à renouveler les questions traitées dans le volume. La première, c'est l'affirmation du facteur religieux (principalement chrétien) comme élément déterminant dans les évolutions sociopolitiques et les enjeux diplomatiques des régions concernées entre la guerre de Crimée et la dissolution de l'Empire ottoman. La seconde, d'ordre méthodologique, est la volonté de penser la complexité en sortant du cadre des histoires nationales et confessionnelles pour postuler d'une part, qu'il y a des relations d'ordre structurel ou conjoncturel entre les situations qui se jouent sur les différents théâtres envisagés ici, des Balkans au Proche-Orient, et d'autre part qu'il faut prendre en compte non seulement les interactions entre diverses confessions présentes sur un 
même terrain, mais aussi la diversité de positionnement entre les acteurs appartenant à une seule confession - cette dernière n'étant pas traitée comme une entité homogène. On pourrait ajouter que les contributions du volume jouent sur la variation des échelles chronologiques, entre le temps long et lent des institutions et des doctrines, qui caractérise les Églises, et le temps haletant de l'événement, dans une période de bouleversements complets, rapides et imprévus. Elles jouent aussi sur les échelles spatiales, en tentant pour la plupart de montrer les interactions entre faits divers locaux et politiques nationales ou internationales, ou de partir des acteurs individuels pour saisir la complexité des évolutions générales. Cette histoire qui tient compte du contexte et de la place de l'acteur dans un réseau doit souvent recourir à une minutieuse histoire événementielle, qui, à certains moments, frise la démarche historicisante. Comme souvent dans les ouvrages collectifs, la lecture des articles éveille chez le lecteur des élans de comparaison et de rapprochement, mais le recueil lui-même n'offre que partiellement des éléments de synthèse entre les différents théâtres évoqués.

2 La période étudiée correspond à la fin du concert des nations institué par le Congrès de Vienne, qui donnait aux puissances une responsabilité collective dans le maintien de l'ordre international. La rivalité entre puissances, qui va en s'exacerbant à partir de la guerre de Crimée, offre plus de marge de manœuvre aux petites nations balkaniques. L'Empire ottoman pour sa part doit s'adapter aux nouvelles conditions économiques, militaires et diplomatiques. Pour ce faire, il tente de réformer son système fiscal et administratif par une politique de centralisation et d'homogénéisation, qui touche aussi l'organisation des communautés (millets). Les chefs ecclésiastiques sont intégrés dans le schéma administratif de l'Empire. Le clergé local participe à l'administration et siège dans les conseils régionaux et locaux, bien que, dans le même temps, le développement rapide des œuvres sociales et scolaires assure aux laïcs une place plus active dans les communautés (Kostas Kostis). En même temps, l'offre confessionnelle s'élargit, avec l'implantation de missionnaires catholiques et protestants qui proposent aux individus ou aux groupes des alternatives aux Églises locales traditionnelles.

Des communications s'attachent à analyser les conséquences de ces transformations sur des situations précises, à l'échelle la plus rapprochée, privilégiant une lecture " buttom-up ». À Ghazir, un village du Mont Liban (Chantal Verdeil), comme à Malko Tărnovo, en Thrace, aujourd'hui en Bulgarie (Andreas Lyberatos), une unité de vie locale est traversée par des divisions entre quartiers et entre partisans de leaders rivaux, dont la place est menacée par les changements économiques et politiques introduits par les réformes ottomanes. Mais ces circonstances locales interfèrent avec un contexte plus large, car les protagonistes peuvent chercher des protecteurs et des arbitres extérieurs (missionnaires, consuls de France, fonctionnaires ottomans). Un conflit de cette nature peut alors se confessionnaliser et s'ethniciser : à Malko Tărnovo, le parti des "pauvres» touchés par la prolétarisation, se tourne vers le catholicisme uniate, contre les notables auxquels l'évêque orthodoxe appartient. Les premiers apparaissent alors comme "Bulgares", tandis que les seconds sont assimilés aux «Grecs ». À l'ère des réformes, l'ambiguïté n'était plus tolérée : à la sortie du conflit, il a fallu s'enregistrer dans l'état civil en tant que catholique ou en tant qu'orthodoxe.

4 En Bulgarie encore, la population musulmane ne fut pas contrainte à l'exil après l'indépendance, comme ce fut le cas en Grèce ou en Serbie. Toutefois, s'intégrer à la nation bulgare, construite sur l'anti-ottomanisme, n'avait rien d'évident pour cette 
minorité, généralement abordée dans l'historiographie comme victime du nouvel ordre politique et institutionnel. Milena. B. Methodieva entend au contraire montrer ici que les musulmans furent aussi des acteurs n'attendant pas leur salut exclusivement d'Istanbul dans le jeu marquant la phase de construction de l'État bulgare. Dans les années 1890 , une intelligentsia musulmane prit la tête d'un mouvement qui prônait une réforme autochtone, appuyée sur une presse musulmane locale et liée à l'organisation des Jeunes Turcs, dont la Bulgarie était une base arrière qui accueillait nombre d'exilés d'Istanbul. Alors que l'appartenance religieuse pouvait s'ethniciser dans d'autres contextes, dans le cas des musulmans bulgares, qui ne formaient pas un groupe homogène, mais étaient au contraire ethniquement très bigarrés, la religion fut utilisée comme un marqueur identitaire fort et un facteur de mobilisation de la minorité, auxquels les Jeunes Turcs ne renoncèrent pas, tout en s'attaquant aux élites religieuses traditionnelles, accusées d'ignorance et de complaisance envers le régime bulgare ou celui du sultan Abdulhamit II.

Dans le cas du Kosovo de la fin de l'Empire ottoman (Eva A. Frantz), une classification ethnique stricte en Serbes orthodoxes et Albanais musulmans, partagés par une haine réciproque, serait anachronique. Si les albanophones musulmans étaient les plus nombreux, face aux slavophones orthodoxes, la mosaïque ethnique était plus complexe. Ethnies et confessions ne se recoupaient qu'imparfaitement, et les distinctions sociales jouaient leur rôle. D'autre part, les structures tribales et le droit coutumier primaient dans une grande partie des campagnes, où les notions d'honneur et de virilité, symbolisées par le port du fusil, maintenaient un certain degré de violence, indépendamment de l'appartenance confessionnelle. Toutefois, l'introduction des réformes ottomanes, l'ingérence des consuls étrangers en tant que "protecteurs » des chrétiens, et l'expulsion des musulmans des territoires voisins, avaient pour effet de faire monter de degré cette violence endémique ou quotidienne et de lui donner un contenu ethnique et confessionnel plus net. La rumeur, générant peur et agressivité, joua partout un rôle dans la montée des affrontements. Le mécanisme décrit ici présente des similitudes avec celui qui déboucha sur les massacres de chrétiens au Mont Liban et à Damas en 1860 et avec l'enchaînement de violence évoqué ici par Nikos Andriotis dans son article consacré à la Crète.

L'implication directe des Puissances dans la région contribua également à l'exacerbation du confessionnalisme et des nationalismes sur des bases ethniques. L'Autriche-Hongrie, la Grande-Bretagne et l'Italie prirent sous leur contrôle des territoires et $\mathrm{y}$ expérimentèrent directement la gestion d'une «mosaïque ethnoconfessionnelle ». La volonté d'introduire la modernité politique libérale dans les territoires sous administration coloniale entra en contradiction avec la nécessité d'assurer la représentativité des communautés en tant que groupes, ce qui amena à délimiter strictement leurs frontières confessionnelles. De plus, le principe réaliste de "diviser pour régner" et la précaution de brider l'émergence d'une véritable citoyenneté l'emportèrent souvent dans l'action des gouvernants coloniaux (A. Anastassiadis). L'exemple de Chypre sous l'occupation britannique (1874-1884) illustre, d'une part, ce croisement entre une logique impériale ottomane, fondée sur la gestion des communautés, et une logique coloniale britannique favorisant la sécularisation, d'autre part, la capacité d'adaptation des forces locales à la nouvelle donne politique et institutionnelle dont elles devinrent des actives collaboratrices. Tandis que les membres de la millet grecque s'identifièrent au "peuple " grec, les musulmans revendiquèrent un traitement égalitaire en tant que « peuple» musulman. 
Une division territoriale de l'île entre les deux «peuples " s'amorça. La construction d'une nation chypriote sur la base d'une citoyenneté non confessionnelle fut ainsi condamnée à l'échec (Sia Anagnostopoulou).

7 L'article d'Ines Sabotič montre comment, en Bosnie-Herzégovine sous gouvernement autrichien, des Croates inventèrent la narration historique du bogomilisme en vue d'intégrer les musulmans dans le récit national croate. Mais la tendance séculariste du nationalisme croate se heurtait à la tendance cléricale, qui liait la nation à la religion catholique. Les musulmans pour leur part suivaient leur propre agenda. L'introduction d'une représentation parlementaire fondée sur une base ethnoconfessionnelle aboutit à une coalition islamo-serbe, avant de déboucher sur l'idée de "Yougoslavie » fondée sur une coalition « chrétienne » entre Serbes et Croates.

8 L'intervention humanitaire internationale en faveur d'une minorité ethnique ou confessionnelle maltraitée, dont la période eut la primeur, aboutit elle aussi à renforcer les clivages ethnoconfessionnels, débouchant même parfois sur le "nettoyage ethnique ». En Crète, comme au Mont Liban, la question sociale fut confessionnalisée et ethnicisée par l'appel à l'opinion internationale. Les sentiments d'espoir ou au contraire d'insécurité suscités par l'arrivée des nouvelles des autres provinces ottomanes débouchaient sur des violences de plus en plus incontrôlées. L'envoi par les puissances d'un corps expéditionnaire à vocation humanitaire ne semblait destiné qu'à favoriser la composante chrétienne. En Crète, malgré des dispositions constitutionnelles pour protéger la minorité musulmane, la part de celle-ci dans la population de l'île diminua de façon drastique après l'établissement d'un statut d'autonomie, tandis qu'on assistait à une redistribution des biens fonciers en faveur des chrétiens (N. Andriotis).

9 La seconde et la quatrième partie de l'ouvrage partent plutôt du rôle des acteurs étrangers, sur le "théâtre levantin des impérialismes » (Patrick Cabanel). L'article de Dominique Trimbur montre comment la conjoncture politique interne permit progressivement aux catholiques allemands de s'implanter de façon autonome en Palestine, en s'émancipant de la tutelle de la France, «protectrice » des catholiques, et en servant d'instrument à la politique expansionniste du Kaiser. L'investissement russe en Palestine présente une certaine concomitance avec celui des Allemands, à l'égard duquel il fit preuve de mimétisme (Elena Astafieva). La Société impériale orthodoxe de Palestine devint «un lieu de rencontre et de croisement entre le savoir russe, la diplomatie impériale, et la religion orthodoxe ", mais une opposition se dessina entre partisans d'une palestinologie sécularisée et partisans d'un usage identitaire et politique de la recherche archéologique en Terre sainte, au service d'une certaine conception de l'orthodoxie.

10 La politique française, et notamment son « influence » à travers l'action éducative des congrégations catholiques, se révèle également complexe et contradictoire. La contribution de Jérôme Bocquet offre des éléments pour déconstruire le contenu impérialiste de la «mission civilisatrice de la France ». L'action de cette dernière se caractérise par le fait qu'elle ne gère pas directement des territoires, mais qu'elle s'appuie sur un réseau consulaire et missionnaire, qui la rend omniprésente (T. Anastassiadis).

11 L'article de Nadia Danova, qui retrace l'histoire des missions protestantes en Bulgarie, montre que la chronologie, les méthodes et les résultats, dans ce cas particulier, sont assez comparables à ce qu'on observe à la même époque concernant l'implantation 
protestante au Mont Liban ou en Anatolie. D’Égypte aux Balkans, les valeurs " modernes» et le genre de vie diffusés par les missionnaires protestants eurent un impact sans aucune proportion avec le nombre restreint des convertis à l'évangélisme. Le catholicisme romain est de même resté très minoritaire dans l'ère envisagée, mais a exercé une influence non négligeable à travers son dense réseau scolaire et sa capacité d'intervention directe. La contribution de Nathalie Clayer, portant sur les missions jésuites en Albanie, nous rappelle qu'avec des objectifs et des méthodes qui ont peu évolué depuis le XVII siècle, les missionnaires latins cherchaient d'abord à renforcer la conscience confessionnelle des minoritaires catholiques, à travers l'inculcation d'un habitus qui devait les distinguer de leur environnement multiconfessionnel où régnaient des formes de culture (y compris religieuse) partagée.

La Première Guerre mondiale donna lieu à des projets mirifiques, qu'on peut rétrospectivement ranger au rayon des rêves éveillés et des étranges chimères qui ont habité les impérialismes en concurrence au Levant, et dont cet ouvrage donne plusieurs exemples. L'effondrement de l'Empire russe, puis de l'Empire ottoman, nourrit des espoirs insensés de réunification religieuse centrés autour de la prise de possession politique et symbolique de Constantinople, avec le retour de Sainte-Sophie au culte orthodoxe ou catholique. L'Église catholique adopta à l'égard des réfugiés russes à Istanbul, puis des réfugiés anatoliens en Grèce, une "diplomatie de l'assistance " qu'elle avait commencé à expérimenter durant la Première Guerre mondiale. Elle s'accompagnait d'un plan d'action à destination de l'Église russe et de son union avec Rome (L. Pettinaroli) ou d'une tentative de normalisation des rapports avec la Grèce, pour gérer à travers un concordat les affaires de la minorité catholique du pays (Giuseppe M. Croce). Mais, comme le rappelle Claude Prud'homme, le modèle élaboré par l'Église catholique depuis le XVII siècle, d'une occupation méthodique de l'univers habité fondée sur une centralisation garantissant son efficacité, s'est heurté à un autre modèle d'ecclésiologie et à une autre conception de l'organisation du temps et de l'espace, en vigueur dans le christianisme oriental. Un rêve concurrent, dont les prémisses sont déjà perceptibles au $\mathrm{XVII}^{\mathrm{e}}$ siècle, était celui d'une nouvelle oecumène chrétienne, orthodoxe-anglicane, qui s'étendrait de la Californie à la Sibérie, et aurait Constantinople comme centre. Ce rêve avait commencé à prendre consistance dans le rapprochement de prélats orthodoxes avec les anglicans à partir du milieu du XIX siècle, et était en grande partie fondé sur l'hostilité de la Grèce à l'égard des ambitions russes et de "l'uniastime» romain. Il devait se briser sur la défaite grecque face à Mustafa Kemal, mais aussi sur la contradiction entre la vision d'un patriarcat universaliste et supranational d'une part, une conception "byzantine» grecque de l'association entre les pouvoirs régulier et séculier, et une logique impériale multiconfessionnelle britannique. Mais l'idée d'un patriarcat de Constantinople fédérant les orthodoxes au niveau mondial n'est aujourd'hui pas complètement hors de l'actualité. 